\title{
Nonperturbative Coherent Population Trapping: An Analytic Model
}

\author{
V. Delgado and J. M. Gomez Llorente \\ Departamento de Física Fundamental II, \\ Universidad de La Laguna, 38205-La Laguna, Tenerife, Spain
}

\begin{abstract}
Coherent population trapping is shown to occur in a driven symmetric double-well potential in the strong-field regime. The system parameters have been chosen to reproduce the $0^{-} \leftrightarrow 3^{+}$transition of the inversion mode of the ammonia molecule. For a molecule initially prepared in its lower doublet we find that, under certain circumstances, the $3^{+}$level remains unpopulated, and this occurs in spite of the fact that the laser field is resonant with the $0^{-} \leftrightarrow 3^{+}$transition and intense enough so as to strongly mix the $0^{+}$and $0^{-}$ground states. This counterintuitive result constitutes a coherent population trapping phenomenon of nonperturbative origin which cannot be accounted for with the usual models. We propose an analytic nonperturbative model which accounts correctly for the observed phenomenon.
\end{abstract}

42.50.Hz, 42.50.Gy, 03.65.-w

Quantum dynamics in symmetric double-well potentials is important to understand numerous physical and chemical processes. A typical example is the tunneling dynamics of the hydrogen atoms in the inversion mode of the ammonia molecule, which is responsible for the splitting of the vibrational levels [1]. Other examples include electron tunneling in quantum semiconductor structures [2] or intermolecular proton transfer processes [3].

In recent years there has been increasing interest in quantum coherence phenomena displayed by atomic and molecular systems irradiated with strong laser fields [4]. Coherent external fields induce quantum interference effects such as coherent population trapping [5], electromagnetically induced transparency [6] or lasing without inversion [7]. In particular, in connection with the time evolution of a quantum system in a symmetric doublewell potential it has been shown that under certain circumstances an intense laser field can induce coherent tunneling suppression [8]. In this Letter we show that such a system can also exhibit coherent population trapping. This population trapping phenomenon is nonperturbative in nature and cannot be accounted for with the usual models. We propose an analytically solvable nonperturbative model which accounts correctly for the essential features of the observed phenomenon.

Specifically, we consider a symmetric quartic doublewell potential strongly driven by a linearly polarized laser field. After appropriate scaling the corresponding dimensionless Hamiltonian reads

$$
H=\frac{P^{2}}{2}-\frac{X^{2}}{4}+\frac{X^{4}}{64 \alpha}-\lambda X \cos (\tau)
$$

where the coupling constant $\lambda$ is proportional to the laser field amplitude $\mathbf{E}_{0}$ and $\tau=\omega_{\mathrm{L}} t$ with $\omega_{\mathrm{L}}$ being the laser frequency. The dimensionless parameter $\alpha$, which gives approximately the number of doublets below the barrier top, has been chosen to be 1.735 . This value reproduces, to a good approximation, the effective potential involved in the inversion mode of the ammonia molecule. The laser frequency has been tuned to the $0^{-} \leftrightarrow 3^{+}$vibrational transition and its intensity satisfies $\lambda\left\langle 0^{+}|X| 0^{-}\right\rangle=\mathbf{E}_{0} \mu_{12}=0.35 \pi \omega_{\mathrm{L}}$ where $\mu_{12}$ is the dipole matrix element between the ground states $\left|0^{+}\right\rangle$and $\left|0^{-}\right\rangle$, and all quantities are assumed to be dimensionless.

Transitions $0^{+} \leftrightarrow 0^{-}$and $0^{-} \leftrightarrow 3^{+}$are dipole allowed whereas the $0^{+} \leftrightarrow 3^{+}$transition is forbidden. Therefore, in the weak-field regime $\left(\mathbf{E}_{0} \mu_{12} / \omega_{\mathrm{L}}, \Delta_{0} / \omega_{\mathrm{L}} \ll 1\right.$ with $\Delta_{0}$ being the energy splitting of the lower doublet) and for a laser field tuned to the $0^{-} \leftrightarrow 3^{+}$transition, one expects the upper level to be populated or not depending on whether the molecule is initially prepared in the $\left|0^{-}\right\rangle$ or $\left|0^{+}\right\rangle$state, respectively. The laser intensity considered above, however, corresponds to the strong-field regime $\left(\mathbf{E}_{0} \mu_{12} \approx \omega_{\mathrm{L}}\right)$. Under these circumstances the two lower levels become strongly mixed and the $\left|0^{-}\right\rangle$state becomes highly populated. One then would expect the upper level to be populated irrespective of the fact that the molecule be initially prepared in the $\left|0^{-}\right\rangle$or $\left|0^{+}\right\rangle$state.

Fig. 1a shows the evolution of the populations for an ammonia molecule initially prepared in its ground state $\left|0^{+}\right\rangle$. These results have been obtained numerically by direct integration of the Schrödinger equation. We have included the 20 lowest-lying levels, which guarantees convergence. As is apparent from the figure, the upper level $\left|3^{+}\right\rangle$remains always unpopulated [curve (3)], and this occurs despite the fact that the $\left|0^{-}\right\rangle$state becomes highly populated [curve (2)] and the laser field directly connects this latter state with the upper level. This figure also shows that the total population in the lower doublet remains always close to unity [curve (1)]. Thus, under the action of the driving field the initial population oscillates rapidly between $\left|0^{+}\right\rangle$and $\left|0^{-}\right\rangle$while it remains trapped in the lower doublet. This counterintuitive result represents a coherent population trapping phenomenon of nonperturbative nature which cannot be accounted for with the usual models.

The case of an ammonia molecule prepared initially in $\left|0^{-}\right\rangle$is considered in Fig. 1b. This figure shows the time evolution of both the population of the upper level $\left|3^{+}\right\rangle$[curve (3)] and the total population of the lower doublet [curve (1)]. As before the initial population os- 
cillates very rapidly between $\left|0^{-}\right\rangle$and $\left|0^{+}\right\rangle$(not shown for clarity). Now, however, a periodic population transfer between the lower doublet and the upper level takes place on a different timescale. In fact, apart from the rapid oscillations of the upper level population (which originates from population transfers to levels adjacent to $\left|3^{+}\right\rangle$, as a detailed numerical analysis reveals) the behavior of the system in this nonperturbative regime resembles that of the corresponding weak-field regime.
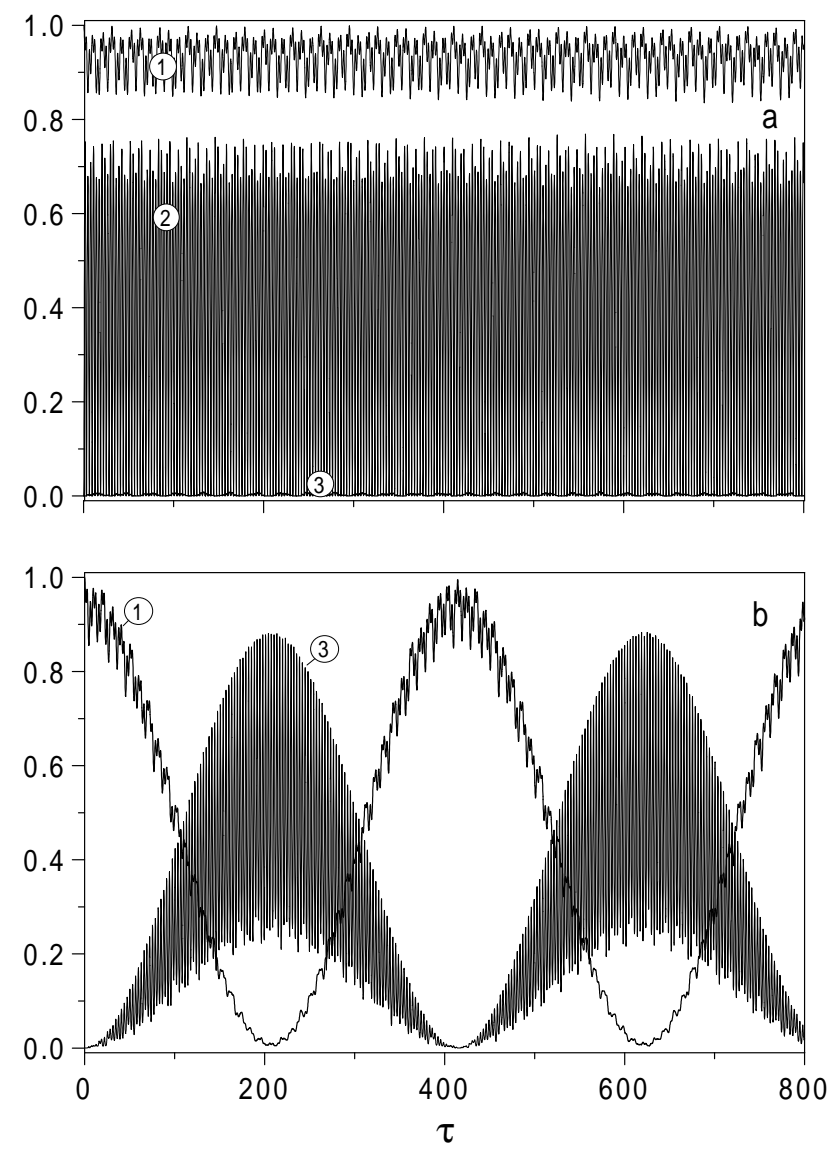

FIG. 1. Populations vs. $\tau=\omega_{\mathrm{L}} t$ for an ammonia molecule initially prepared in: (a) the $\left|0^{+}\right\rangle$state; and (b) the $\left|0^{-}\right\rangle$state. Curves (1) give the total population of the lower doublet; curve (2) gives the population of the $\left|0^{-}\right\rangle$state; and curves (3) give the population of the upper level $\left|3^{+}\right\rangle$.

The above results pose two intriguing questions: i) Why does the population become trapped when the molecule is initially prepared in its ground state? and ii) Why does the system behave essentially in a similar way both in the nonperturbative strong-field regime and in the weak-field regime? In what follows, we propose an analytic nonperturbative three-level model which can give an answer to these questions.

The most directly involved states, $\left|0^{+}\right\rangle,\left|0^{-}\right\rangle$and $\left|3^{+}\right\rangle$, will be denoted $|1\rangle,|2\rangle$ and $|3\rangle$, respectively. The energy splitting of the lower doublet is $\Delta_{0}$, and $\omega_{3}$ denotes the energy of the upper level. The system Hamiltonian is

$$
\begin{aligned}
H=\frac{\Delta_{0}}{2}\left(\sigma_{22}-\sigma_{11}\right) & +\omega_{3} \sigma_{33}-\Omega_{12} \cos (\tau)\left(\sigma_{12}+\sigma_{21}\right) \\
& -\Omega_{23} \cos (\tau)\left(\sigma_{23}+\sigma_{32}\right),
\end{aligned}
$$

where $\hbar \equiv 1 ; \sigma_{i j} \equiv|i\rangle\langle j|$; and $\Omega_{i j} \equiv \mathbf{E}_{0} \mu_{i j}$ with $\mu_{i j}$ being the dipole matrix elements between $|i\rangle$ and $|j\rangle$.

The most rapidly oscillating terms can be absorbed by performing the unitary transformation

$$
U(\tau)=\exp \left[-i \frac{\Omega_{12}}{\omega_{\mathrm{L}}}\left(\sigma_{12}+\sigma_{21}\right) \sin (\tau)+i \sigma_{33} \tau\right],
$$

which leads to the transformed Hamiltonian

$$
\begin{aligned}
H^{\prime} & =\left(\Delta_{0} / 2\right)\left\{\cos [2 \phi(\tau)]\left(\sigma_{22}-\sigma_{11}\right)\right. \\
& \left.+i \sin [2 \phi(\tau)]\left(\sigma_{21}-\sigma_{12}\right)\right\}+\left(\omega_{3}-\omega_{\mathrm{L}}\right) \sigma_{33} \\
& -\Omega_{23} \cos (\tau)\left\{e ^ { - i \tau } \left(\cos [\phi(\tau)] \sigma_{23}\right.\right. \\
& \left.\left.-i \sin [\phi(\tau)] \sigma_{13}\right)+ \text { h.c. }\right\}
\end{aligned}
$$

with $\phi(\tau)=\left(\Omega_{12} / \omega_{\mathrm{L}}\right) \sin (\tau)$. Next, we expand the time dependent coefficients of $H^{\prime}$ in Fourier series, which allows us to separate the Hamiltonian into a dominant constant contribution $H_{0}^{\prime}$ and a time-dependent part $\Delta H^{\prime}(\tau)$. Then, substitution of $H^{\prime}$ into the evolution operator of the system shows that when the driving field is quasiresonant with the $|2\rangle \leftrightarrow|3\rangle$ transition and both the energy difference $\Delta_{0}$ and the Rabi frequency $\Omega_{23}$ are small in comparison with the laser frequency, $\Delta H^{\prime}(\tau)$ becomes a small, rapidly oscillating perturbation which can be safely neglected. More generally, it can be shown that in the strong-field regime $\left(\Omega_{12} / \omega_{\mathrm{L}} \gtrsim 1\right)$ and for a quasiresonant laser field, $\Delta H^{\prime}(\tau)$ becomes negligible whenever $\Delta_{0} / \omega_{\mathrm{L}}, \Omega_{23} / \omega_{\mathrm{L}} \ll \sqrt{\Omega_{12} / \omega_{\mathrm{L}}}$. (In our case, $\Delta_{0} / \omega_{\mathrm{L}}=3.28 \times 10^{-4}, \Omega_{23} / \omega_{\mathrm{L}}=0.23$, and $\Omega_{12} / \omega_{\mathrm{L}}=$ 1.10.) Under these circumstances, the dynamical evolution of the system is governed by the Hamiltonian

$H^{\prime} \equiv \frac{\Delta_{0}^{\mathrm{R}}}{2}\left(\sigma_{22}-\sigma_{11}\right)+\left(\omega_{3}-\omega_{\mathrm{L}}\right) \sigma_{33}-\frac{\Omega_{23}^{\mathrm{R}}}{2}\left(\sigma_{23}+\sigma_{32}\right)$,

where the renormalized energy difference $\Delta_{0}^{\mathrm{R}}$ and Rabi frequency $\Omega_{23}^{\mathrm{R}}$ are field-dependent quantities defined as $\Delta_{0}^{\mathrm{R}}=\Delta_{0} J_{0}\left(2 \Omega_{12} / \omega_{\mathrm{L}}\right)$ and $\Omega_{23}^{\mathrm{R}}=$ $2 \omega_{\mathrm{L}}\left(\Omega_{23} / \Omega_{12}\right) J_{1}\left(\Omega_{12} / \omega_{\mathrm{L}}\right)$, with $J_{n}$ being the $\mathrm{n}^{\text {th }}$-order Bessel function. The Schrödinger equation associated with the above Hamiltonian can be readily solved analytically, and after transforming back one obtains the following nonperturbative general solution

$$
|\Psi(\tau)\rangle=C_{1}(\tau)|1\rangle+C_{2}(\tau)|2\rangle+C_{3}(\tau)|3\rangle
$$

where

$$
\begin{aligned}
& C_{1}(\tau)=C_{1}^{\prime}(\tau) \cos \phi(\tau)+i C_{2}^{\prime}(\tau) \sin \phi(\tau) \\
& C_{2}(\tau)=C_{2}^{\prime}(\tau) \cos \phi(\tau)+i C_{1}^{\prime}(\tau) \sin \phi(\tau)
\end{aligned}
$$




$$
C_{3}(\tau)=C_{3}^{\prime}(\tau) e^{-i \tau}
$$

and the $C_{i}^{\prime}(\tau)$, which are the probability amplitudes associated with the Hamiltonian (5), are given by

$$
\begin{gathered}
C_{1}^{\prime}(\tau)=C_{1}^{\prime}(0) e^{i \frac{\Delta_{0}^{\mathrm{R}}}{2 \omega_{\mathrm{L}}} \tau} \\
C_{2}^{\prime}(\tau)=\left\{C_{2}^{\prime}(0) \cos \left(\frac{\Omega^{\mathrm{R}}}{2 \omega_{\mathrm{L}}} \tau\right)+\frac{i}{\Omega^{\mathrm{R}}}\left(C_{2}^{\prime}(0) \delta^{\mathrm{R}}\right.\right. \\
\left.\left.+C_{3}^{\prime}(0) \Omega_{23}^{\mathrm{R}}\right) \sin \left(\frac{\Omega^{\mathrm{R}}}{2 \omega_{\mathrm{L}}} \tau\right)\right\} e^{-\frac{i}{2 \omega_{\mathrm{L}}}\left(\delta^{\mathrm{R}}+\Delta_{0}^{\mathrm{R}}\right) \tau} \\
C_{3}^{\prime}(\tau)=\left\{C_{3}^{\prime}(0) \cos \left(\frac{\Omega^{\mathrm{R}}}{2 \omega_{\mathrm{L}}} \tau\right)-\frac{i}{\Omega^{\mathrm{R}}}\left(C_{3}^{\prime}(0) \delta^{\mathrm{R}}\right.\right. \\
\left.\left.-C_{2}^{\prime}(0) \Omega_{23}^{\mathrm{R}}\right) \sin \left(\frac{\Omega^{\mathrm{R}}}{2 \omega_{\mathrm{L}}} \tau\right)\right\} e^{\frac{i}{2 \omega_{\mathrm{L}}}\left[\delta^{\mathrm{R}}-2\left(\omega_{3}-\omega_{\mathrm{L}}\right)\right] \tau}
\end{gathered}
$$

where $\delta^{\mathrm{R}}=\omega_{3}-\Delta_{0}^{\mathrm{R}} / 2-\omega_{\mathrm{L}}$ is the renormalized detuning and $\Omega^{\mathrm{R}}=\sqrt{\left(\Omega_{23}^{\mathrm{R}}\right)^{2}+\left(\delta^{\mathrm{R}}\right)^{2}}$ is the renormalized generalized-Rabi-frequency. The physical content of the above solution becomes more transparent by considering the extended Hilbert space of $\tau$-periodic state vectors [9]. In fact, the basis $\left\{\left|i^{\prime}(\tau)\right\rangle\right\}$ with $\left|i^{\prime}(\tau)\right\rangle \equiv U^{+}(\tau)|i\rangle$ turns out to be the natural basis to express $|\Psi(\tau)\rangle$

$$
|\Psi(\tau)\rangle=C_{1}^{\prime}(\tau)\left|1^{\prime}(\tau)\right\rangle+C_{2}^{\prime}(\tau)\left|2^{\prime}(\tau)\right\rangle+C_{3}^{\prime}(\tau)\left|3^{\prime}(\tau)\right\rangle
$$

As this expression reflects, the dynamical evolution of the probability amplitudes corresponding to the renormalized $\left\{\left|i^{\prime}(\tau)\right\rangle\right\}$ states is governed by the Hamiltonian $H^{\prime}$ of Eq. (5). Such Hamiltonian has the same form as the original Hamiltonian (2) in the limit $\Omega_{12} \rightarrow 0$ (in the rotating wave approximation and in the frame rotating with the laser frequency). Therefore, the theory is renormalizable in the sense that when analyzed in terms of the $\left\{\left|i^{\prime}(\tau)\right\rangle\right\}$ states, the nonperturbative effects of the radiation field on the dynamical evolution of the system can be absorbed into the renormalized splitting $\Delta_{0}^{\mathrm{R}}$ and Rabi frequency $\Omega_{23}^{\mathrm{R}}$, in such a way that the system evolves obeying the same Hamiltonian as that of the weak-field regime in the rotating wave approximation. In fact, the general solution (9) is valid both in the (perturbative) weak-field regime $\left(\Omega_{12} / \omega_{\mathrm{L}}, \Delta_{0} / \omega_{\mathrm{L}} \ll 1\right)$ and in the (nonperturbative) strong-field regime $\left(\Omega_{12} / \omega_{\mathrm{L}} \gtrsim 1\right)$.

As Eq. (8a) shows, under the action of the coherent external field, the $\left|1^{\prime}(\tau)\right\rangle$ state decouples and all the population that is initially in state $|1\rangle$ becomes trapped in $\left|1^{\prime}(\tau)\right\rangle$. For a system prepared initially in its ground state this implies, in particular, that the upper level $|3\rangle$ remains always unpopulated. This occurs in spite of the fact that the initial population oscillates very rapidly between the $|1\rangle$ and $|2\rangle$ levels, and the latter is directly coupled to $|3\rangle$ via a laser field tuned to the $2 \leftrightarrow 3$ transition. This is a coherent population trapping phenomenon of nonperturbative origin.
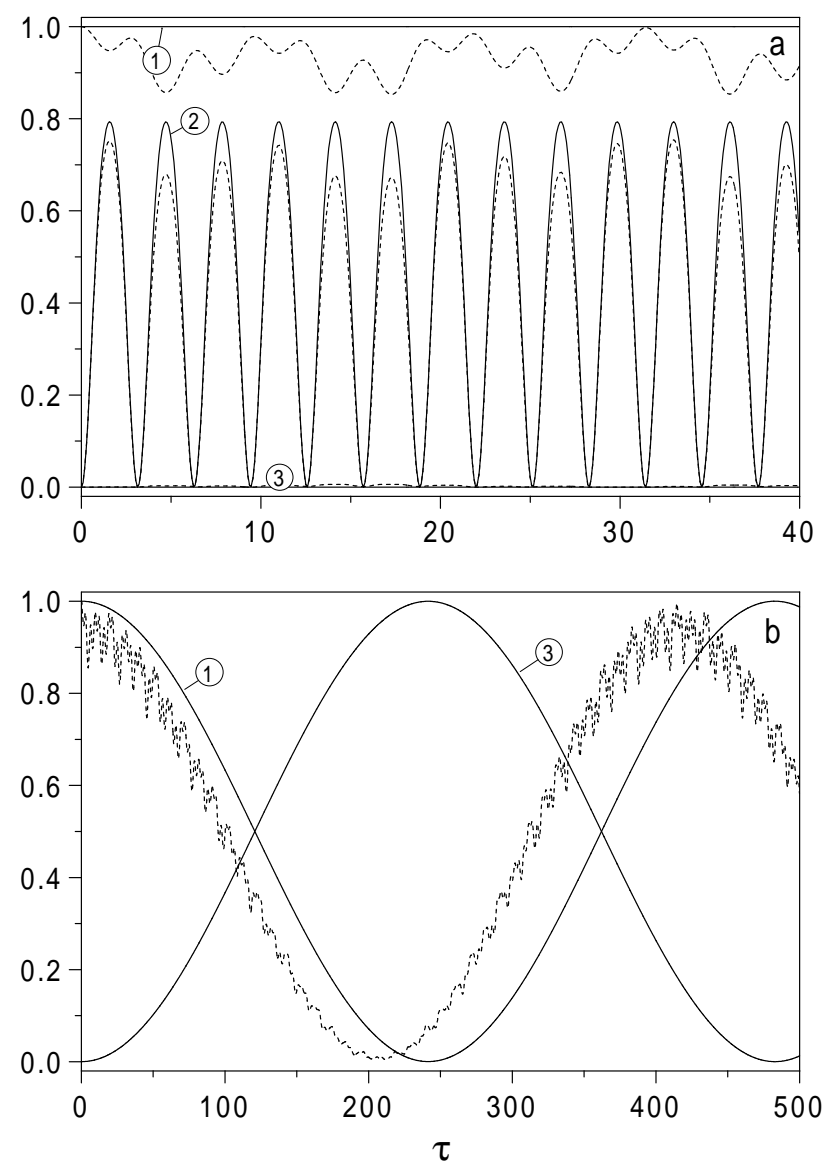

FIG. 2. Theoretical predictions for the same situations considered in Fig. 1. For comparison purposes, along with the analytical results (solid lines) the corresponding exact numerical results have been plotted again (dotted lines).

On the other hand, when the molecule is prepared in $\tau=0$ in the state $|2\rangle$, the population difference between the upper level and the lower doublet oscillates in time as

$$
W(\tau)=-\cos \left(\frac{\Omega^{\mathrm{R}}}{\omega_{\mathrm{L}}} \tau\right)-2\left(\frac{\delta^{\mathrm{R}}}{\Omega^{\mathrm{R}}}\right)^{2} \sin ^{2}\left(\frac{\Omega^{\mathrm{R}}}{2 \omega_{\mathrm{L}}} \tau\right)
$$

Fig. 2 shows the theoretical predictions of our model for the same situations considered in Fig. 1. Fig. 2a corresponds to an ammonia molecule prepared initially in its ground state. In this case, the populations $\rho_{i i}(\tau)$ of the molecular states $\{|i\rangle\}$ are predicted to be

$$
\rho_{22}(\tau)=\sin ^{2}\left(\frac{\Omega_{12}}{\omega_{\mathrm{L}}} \sin \tau\right), \quad \rho_{33}(\tau)=0
$$

and $\rho_{11}(\tau)=1-\rho_{22}(\tau)$. Thus, the population of the lower doublet, which under these circumstances coincides with that of the renormalized state $\left|1^{\prime}(\tau)\right\rangle$, remains always equal to one [curve (1)] and the upper level remains unpopulated [curve (3)], in good agreement with the numerical results (dotted lines). Fig. 2a also compares the analytical result for the population of level $|2\rangle$ [curve (2)] with the corresponding exact numerical result. 
On the other hand, Fig. 2b shows the evolution of the populations for a molecule prepared at $\tau=0$ in state $|2\rangle$. In this case the population of the lower doublet coincides with that of the renormalized state $\left|2^{\prime}(\tau)\right\rangle$ and, according to our model, oscillates in time as [curve (1)]

$\rho_{11}(\tau)+\rho_{22}(\tau)=\cos ^{2}\left(\frac{\Omega^{\mathrm{R}}}{2 \omega_{\mathrm{L}}} \tau\right)+\left(\frac{\delta^{\mathrm{R}}}{\Omega^{\mathrm{R}}}\right)^{2} \sin ^{2}\left(\frac{\Omega^{\mathrm{R}}}{2 \omega_{\mathrm{L}}} \tau\right)$,

while the population of the upper level behaves as $\rho_{33}(\tau)=1-\rho_{11}(\tau)-\rho_{22}(\tau)$ [curve $(3)$ ]. These results are in good qualitative agreement with the corresponding numerical results. The main discrepancy between Figs. $2 \mathrm{~b}$ and $1 \mathrm{~b}$ comes from the rapid oscillatory behavior of the upper level population. As already mentioned, it can be shown that this discrepancy, which decreases as the laser intensity does, originates from population transfers to levels adjacent to the $3^{+}$level, which now have a more significant contribution. In fact, if the numerical problem is restricted to the three levels most directly involved then numerical and analytical results become indistinguishable on the scale of the figures. Our three-level model already captures the essential features of the system and enables us to understand the dominant behavior of the populations in terms of a nonperturbative coherent population trapping phenomenon. The upper level remains unpopulated when the molecule is initially prepared in its ground state because such configuration corresponds to an initial preparation in the trapping state $\left|1^{\prime}(\tau)\right\rangle$.

Next, we will analyze the influence of dissipation on the coherent population trapping phenomenon previously found. Spontaneous emission effects can be conveniently incorporated by assuming that the upper level decays radiatively into state $|2\rangle$ with an effective spontaneous emission rate $\Gamma$. The dynamics of the system is now described in terms of the density operator $\rho(t)$ which obeys the usual master equation (in which we have retained nonsecular terms). By performing the unitary transformation (3) one obtains a transformed master equation for the density operator $\rho^{\prime}(t)=U(t) \rho(t) U^{+}(t)$, which, within the range of validity of our model, leads to the following equations of motion governing the time evolution of populations and coherences:

$$
\begin{gathered}
\dot{\rho}_{11}^{\prime}=\frac{\Gamma}{2}\left(1-\Lambda_{0}\right) \rho_{33}^{\prime} \\
\dot{\rho}_{22}^{\prime}=i \Omega_{23}^{\mathrm{R}}\left(\rho_{32}^{\prime}-\rho_{23}^{\prime}\right)+\frac{\Gamma}{2}\left(1+\Lambda_{0}\right) \rho_{33}^{\prime} \\
\dot{\rho}_{12}^{\prime}=i \Delta_{0}^{\mathrm{R}} \rho_{12}^{\prime}-i \Omega_{23}^{\mathrm{R}} \rho_{13}^{\prime} \\
\dot{\rho}_{13}^{\prime}=i(13 \mathrm{~b}) \\
\end{gathered}
$$

$$
\dot{\rho}_{23}^{\prime}=i \delta^{\mathrm{R}} \rho_{23}^{\prime}-i \Omega_{23}^{\mathrm{R}}\left(\rho_{22}^{\prime}-\rho_{33}^{\prime}\right)-\frac{\Gamma}{2}\left(\rho_{23}^{\prime}-\frac{1}{2} \Lambda_{2} \rho_{32}^{\prime}\right)
$$

with $\Lambda_{n} \equiv J_{n}\left(2 \Omega_{12} / \omega_{\mathrm{L}}\right)(n=0,2) ; \rho_{i j}^{\prime}=\left\langle i\left|\rho^{\prime}(t)\right| j\right\rangle=$ $\left\langle i^{\prime}(t)|\rho(t)| j^{\prime}(t)\right\rangle$; and $\rho_{j i}^{\prime}=\rho_{i j}^{\prime *}$.

As Eq. (13a) reflects, whenever the Rabi frequency $\Omega_{12}$ coupling the two lower-lying states is nonzero, the upper state $|3\rangle$ remains unpopulated in the steady state regardless of the initial preparation. As a consequence, in the steady state the molecular population becomes trapped in the lower doublet and the fluorescence from level $|3\rangle$ vanishes. This behavior, which is in sharp contrast with the well-known behavior of the system in the $\Omega_{12}=0$ limit, is typical of systems exhibiting coherent population trapping and has its origin in quantum interferences involving the two lower-lying levels [5].

It is not hard to see from the above equations that, for arbitrary external fields ( state population of $\left|2^{\prime}(t)\right\rangle$ also vanishes so that all of the population becomes trapped in the steady state in $\left|1^{\prime}(t)\right\rangle$ irrespective of the initial preparation.

In conclusion, we have shown that coherent population trapping can occur in the nonperturbative regime and have proposed an analytically solvable nonperturbative three-level model which enables us to understand the observed phenomenon. Although we have presented results for only one field intensity, essentially the same behavior, in good agreement with our analytic model, occurs in the parameter range $0.1 \pi \lesssim \Omega_{12} / \omega_{\mathrm{L}} \lesssim 0.5 \pi$. A detailed account of the model will be given elsewhere.

This work has been supported by MCYT and FEDER under Grant No. BFM2001-3343.

[1] F. Hund, Z. Phys. 43, 803 (1927).

[2] M. Holthaus and D. Hone, Phys. Rev. B 47, 6499 (1993).

[3] A. Oppenländer, Ch. Rambaud, H. P. Trommsdorff, and J. C. Vial, Phys. Rev. Lett. 63, 1432 (1989).

[4] Z. Ficek and H. S. Freedhoff, in Progress in Optics, edited by E. Wolf (Elsevier, Amsterdam, 2000), p. 389.

[5] G. Alzetta, A. Gozzini, L. Moi and G. Orriols, Nuovo Cimento B 36, 5 (1976); E. Arimondo and G. Orriols, Lett. Nuovo Cimento 17, 333 (1976); E. Arimondo, in Progress in Optics, edited by E. Wolf (Elsevier, Amsterdam, 1996), p. 257 and references therein.

[6] S. E. Harris, Phys. Today 50, No. 7, 36 (1997).

[7] O. Kocharovskaya and Ya. I. Khanin, JETP Lett. 48, 630 (1988); S. E. Harris, Phys. Rev. Lett. 62, 1033 (1989); A. Imamoglu, Phys. Rev. A 40, 2835 (1989); M. O. Scully et al., Phys. Rev. Lett. 62, 2813 (1989); G. S. Agarwal, Phys. Rev. A 44, R28 (1991).

[8] F. Grossmann, T. Dittrich, P. Jung, and P. Hänggi, Phys. 
Rev. Lett. 67, 516 (1991); M. Grifoni and P. Hänggi, Phys. Rep. 304, 229 (1998).

[9] H. Sambe, Phys. Rev. A 7, 2203 (1973). 